\title{
Breaks no Tempo em Comportamento Sedentário e Marcadores Cardiometabólicos em Adolescentes
}

\author{
Breaks in Sedentary Time and Cardiometabolic Markers in Adolescents
}

\author{
Natália Maria Mesquita de Lima Quirino, ${ }^{1,2}$ Alcides Prazeres Filho, ${ }^{2,3}$ A Arthur Oliveira Barbosa, ${ }^{2,3}$ Gerfeson

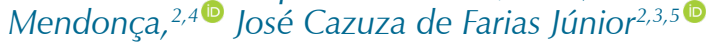 \\ Faculdades Nova Esperança (FACENE), ${ }^{1}$ João Pessoa, PB - Brasil \\ Grupo de Estudos e Pesquisas em Epidemiologia da Atividade Física (GEPEAF), João Pessoa, ${ }^{2}$ PB - Brasil \\ Programa Associado de Pós-Graduação em Educação Física (UPE/UFPB), ${ }^{3}$ João Pessoa, PB - Brasil \\ Centro Universitário Cesmac, ${ }^{4}$ Maceió, AL - Brasil \\ Universidade Federal da Paraíba (UFPB), ${ }^{5}$ João Pessoa, PB - Brasil
}

\section{Resumo}

Fundamento: Interrupções no tempo despendido em comportamento sedentário (breaks) têm sido associadas a melhores níveis de indicadores cardiometabólicos na população adulta. No entanto, em adolescentes, os achados sobre essa associação ainda são conflitantes.

Objetivos: Analisar a associação do número de breaks por dia em comportamento sedentário com marcadores cardiometabólicos e avaliar se ela é moderada pelo estado nutricional e o tempo excessivo em comportamento sedentário em adolescentes.

Métodos: Estudo transversal com 537 adolescentes (52,3\% do sexo feminino), de 10 a 14 anos de idade, de escolas públicas de João Pessoa (PB). O número diário de breaks em comportamento sedentário (>100 counts/minutos) foi mensurado por meio de acelerômetros (Actigraph GT3X+). Os marcadores cardiometabólicos analisados foram: pressão arterial sistólica e diastólica $(\mathrm{mmHg})$, glicose de jejum, colesterol total, triglicerídeos, HDL-c, LDL-c (todos em $\mathrm{mg} / \mathrm{dL}$ ) e índice de massa corporal (IMC) $\left(\mathrm{kg} / \mathrm{m}^{2}\right)$. Utilizou-se a regressão linear para analisar a associação do número de breaks com marcadores cardiometabólicos e avaliar se ela é moderada pelo estado nutricional e o tempo excessivo em comportamento sedentário. O nível de significância de $p<0,05$ foi adotado para todas as análises.

Resultados: O número de breaks por dia se associou negativamente ao IMC ( $(=-0,069 ;$ IC95\%: -0,102; $-0,035)$, mas não aos demais marcadores cardiometabólicos, e essa associação não foi moderada pelo estado nutricional dos adolescentes $(p=0,221)$ e nem pelo tempo excessivo em comportamento sedentário $(p=0,176)$.

Conclusão: A inclusão de breaks no tempo em comportamento sedentário parece contribuir para valores mais baixos do IMC em adolescentes.

Palavras-chave: Adolescente; Sedentarismo; Adiposidade; Marcadores Cardiometabólicos; Pressão Arterial; Colesterol; Glicose; Triglicérides; Comportamento Sedentário.

\footnotetext{
Abstract

Background: The interruption of the time spent in sedentary behavior (breaks) has been associated with better levels of cardiometabolic indicators in the adult population, but in adolescents, further investigations are still needed to confirm these findings.

Objectives: To analyze the association of the number of breaks per day in sedentary behaviors with cardiometabolic markers and whether it was moderated by nutritional status and excessive time on sedentary behavior in adolescents.

Methods: This is a cross-sectional study of 537 adolescents (52.3\% girls), aged between 10 and 14 years, enrolled in public schools in the city of João Pessoa, Paraíba state, Brazil. The number of daily breaks (>100 counts/minutes) in sedentary time was measured by Actigraph GT3X+ accelerometers. The following cardiometabolic markers were analyzed: systolic and diastolic blood pressure ( $\mathrm{mmHg})$, fasting
}

Correspondência: José Cazuza de Farias Júnior •

Universidade Federal da Paraíba, Departamento de Educação Física, Campus I, Cidade Universitária, João Pessoa, Paraíba - Bloco de Pós-Graduação de Fisioterapia e Educação Física - CCS, Grupo de Estudos e Pesquisas em Epidemiologia da Atividade Física (GEPEAF), Sala 20; CEP 58051-900, João Pessoa, PB - Brasil

E-mail: jcazuzajr@hotmail.com, jcazuzajr@gmail.com

Artigo recebido em 18/01/2020, revisado em 02/07/2020, aceito em 16/08/2020

DOI: https://doi.org/10.36660/abc.20200047 
blood glucose levels, total cholesterol, triglycerides, HDL-c, LDL-c (all in $\mathrm{mg} / \mathrm{dL}$ ) and body mass index (BMI) $\left(\mathrm{kg} / \mathrm{m}^{2}\right)$. Linear regression was used to analyze the association between the number of breaks and cardiometabolic markers and whether this association was moderated by nutritional status and excessive time in sedentary behavior. The significance level of $p<0.05$ was adopted for all analyses.

Results: The number of daily breaks was negatively associated with BMI (boys $-\beta=-0.083 ; 95 \% \mathrm{Cl}:-0.132 ;-0.034$ and girls $-\beta=$ $-0.115 ; 95 \% \mathrm{Cl}$ : -0.169; -0.061), but not with the remaining cardiometabolic markers. The number of breaks per day was negatively associated with BMI ( $B=-0.069 ; 95 \% \mathrm{Cl}:-0.102 ;-0.035)$, but not with the other cardiometabolic markers and this association was not moderated by the adolescents' nutritional status $(p=0.221)$, or by excessive time in sedentary behavior $(p=0.176)$.

Conclusions: Including breaks in sedentary time seems to contribute to lower BMI values in adolescents.

Keywords: Adolescent; Sedentarism; Adiposity; Cardiometabolic Markers; Blood Arterial; Cholesterol; Glucose; Triglycerides; Sedentary Behavior.

Full texts in English - http://www.arquivosonline.com.br

\section{Introdução}

Tem sido postulado que o tempo despendido pelos adolescentes em comportamento sedentário - atividades realizadas na posição sentada, reclinada ou deitada, com gasto energético $<1,5 \mathrm{METs}^{1}$ - pode representar um fator de risco para alterações desfavoráveis em marcadores cardiometabólicos ${ }^{2,3}$ e a qualidade de vida relacionada à saúde. ${ }^{4}$ Em função disso, o número de estudos sobre comportamento sedentário e marcadores cardiometabólicos em adolescentes vem crescendo desde a última década. ${ }^{5,6}$

Os efeitos do comportamento sedentário sobre os marcadores cardiometabólicos podem estar relacionados à diminuição da atividade da enzima lipoproteína lipase (LPL), causada pela hipotensão muscular, decorrente da permanência prolongada na posição sentada ou reclinada. ${ }^{7}$ A menor ação da LPL prejudica a captação de triglicerídeos, glicose, insulina e a síntese de lipoproteína de alta densidade (HDL-C). ${ }^{8,9}$ Além disso, o tempo despendido nesses comportamentos está associado à redução na prática de atividade física, sobretudo de intensidade leve, ${ }^{10}$ diminuição do gasto energético total diário, ${ }^{11}$ aumento dos indicadores de gordura corporal ${ }^{2}$ e consumo de alimentos ultraprocessados. . $12,13^{2}$

Estima-se que adolescentes permanecem cerca de 10 horas por dia em comportamento sedentário. ${ }^{14,15}$ e 30,2\% destes passam mais de 8 horas por dia nesse comportamento. ${ }^{16}$ Nesse sentido, a inclusão de interrupções no tempo despendido por dia nesses comportamentos, denominadas breaks, vem sendo considerada como uma das formas de minimizar os efeitos deletérios decorrentes da exposição excessiva e ininterrupta aos comportamentos sedentários. ${ }^{17}$

A incorporação de breaks no tempo sedentário reduz a hipotensão muscular, ${ }^{18}$ elevando a atividade da LPL. ${ }^{19}$ Os breaks também promovem uma elevação no gasto energético total diário devido ao aumento no tempo de prática de atividade física, sobretudo de intensidade leve, ${ }^{20}$ que pode contribuir para um menor acúmulo de gordura corporal ${ }^{21} \mathrm{e}$ melhora nas concentrações de lipoproteínas. ${ }^{22}$

Em adultos, o número de breaks por dia tem sido relacionado com redução na glicemia pós-prandial, ${ }^{21}$ perfil lipídico, ${ }^{23}$ índice de massa corporal $(\mathrm{IMC})^{24}$ e controle da adiposidade. ${ }^{21} \mathrm{Em}$ adolescentes, a quantidade de estudos sobre breaks e marcadores cardiometabólicos ainda é relativamente baixa e com resultados divergentes. . $^{2,5-7,15,25-28}$ Os estudos que identificaram associações significativas entre breaks e marcadores cardiometabólicos nessa população não ajustaram as análises pelo tempo de sono e consumo alimentar; ${ }^{15,26,28}$ foram realizados com adolescentes com excesso de peso ${ }^{27}$ ou histórico familiar de obesidade ${ }^{26}$ e não avaliaram se essa associação era moderada pelo estado nutricional ${ }^{28}$ e/ou tempo excessivo em comportamento sedentário. ${ }^{15,26,28}$

Outra lacuna de conhecimento é se a associação entre o número de breaks e marcadores cardiometabólicos é moderada pelo estado nutricional e/ou tempo sedentário, tendo em vista que o excesso de peso corporal ${ }^{29,30}$ e o tempo excessivo de comportamento sedentário ${ }^{2,6,7}$ estão associados a alterações nos marcadores cardiometabólicos. Dessa forma, a associação entre a realização de breaks no tempo em comportamento sedentário e marcadores cardiometabólicos pode ter diferenças (significância e/ou magnitude) conforme o estado nutricional e/ou do tempo despendido em comportamento sedentário. Sendo assim, este estudo analisou a associação do número de breaks por dia em comportamentos sedentários com marcadores cardiometabólicos, e se era moderada pelo estado nutricional e tempo excessivo em comportamento sedentário em adolescentes.

\section{Métodos}

Estudo transversal que analisou dados referentes ao primeiro ano (2014) do Estudo Longitudinal sobre Comportamento Sedentário, Atividade Física, Hábitos Alimentares e Saúde de Adolescentes (LONCAAFS). A população de referência foi composta por adolescentes de ambos os sexos, de 10 a 14 anos de idade, que estavam matriculados no 6 o ano em escolas da rede pública de ensino da cidade de João Pessoa, Paraíba, Nordeste, Brasil. O Estudo LONCAAFS foi aprovado pelo Comitê de Ética em Pesquisa com Seres Humanos do Centro de Ciências da Saúde da Universidade Federal da Paraíba (Protocolo 240/13).

No presente estudo, foram analisados dados de uma subamostra de adolescentes do Estudo LONCAAFS que utilizou acelerômetros e realizou exame de sangue. Essa escolha foi devido ao número de acelerômetros disponíveis (n = 64), tempo disponível para a coleta de dados e limitação de recursos financeiros. A distribuição da amostra e subamostra levou em consideração a localização da escola na região geográfica no município e o número de alunos matriculados, sendo similar à observada na população de referência. 
Informações sobre o processo de amostragem deste estudo estão apresentadas em detalhes na Figura 1.

A coleta de dados foi realizada no período de fevereiro a junho e de agosto a dezembro de 2014, por equipe treinada, que utilizou um protocolo uniforme de coleta. Foi utilizado um questionário para avaliar variáveis sociodemográficas, horas de sono e consumo alimentar, aplicado em forma de entrevista "face a face". Para coletar os dados sociodemográficos, foram considerados sexo (masculino e feminino), idade em anos completos, cor da pele (parda/morena; preta; branca; amarela; indígena, reagrupadas em "branco" e "não branco"), classe econômica (critérios da Associação Brasileira das Empresas de Pesquisa [ABEP], que agrupa as famílias em A1, $A 2, B 1, B 2, C 1, C 2$, D e E, posteriormente reagrupada em classe $A / B$ [classe mais alta] e classe $C / D / E$ [classe mais baixa] ${ }^{31}$ e escolaridade da mãe (fundamental incompleto, fundamental completo e médio completo ou mais).

As horas de sono foram mensuradas pela seguinte questão: "nos dias de semana e de fim de semana, a que horas você vai dormir e a que horas você acorda?". Para determinar o tempo de sono diário, utilizou-se a média ponderada (horas/ dia), conforme segue: somatório do valor da diferença entre o horário de dormir e de acordar, multiplicado por cinco para os dias de semana, e por dois para os dias de final de semana, dividido por sete. Essa questão apresentou elevado nível de reprodutibilidade (coeficiente de correlação intraclasse [CCI] =0,91; IC95\%: 0,88-0,93).

O consumo de alimentos foi mensurado por meio da aplicação de um recordatório alimentar de 24 horas. ${ }^{32}$ Os adolescentes informaram os alimentos e bebidas consumidos no dia anterior à entrevista, bem como seu meio de preparo, peso e o tamanho das porções. Foi realizada uma replicação em $30 \%$ da amostra para aumentar precisão das estimativas de ingestão dietética. ${ }^{33}$ Os dados foram tabulados no software "Virtual Nutri", e o valor calórico total da dieta foi analisado pela equação da Foodand Nutrition Board of Washinghton. ${ }^{34}$ Neste estudo, foram utilizados os valores de consumo de lipídios (gramas), total de gorduras saturadas (g), colesterol (mg), sódio (mg) e fibras (g).

A medida de massa corporal foi aferida em balança digital, com precisão de 100 gramas, da marca Techline ${ }^{\circledR}$, e a estatura realizada em estadiômetro portátil da marca Sanny ${ }^{\circledR}$. As

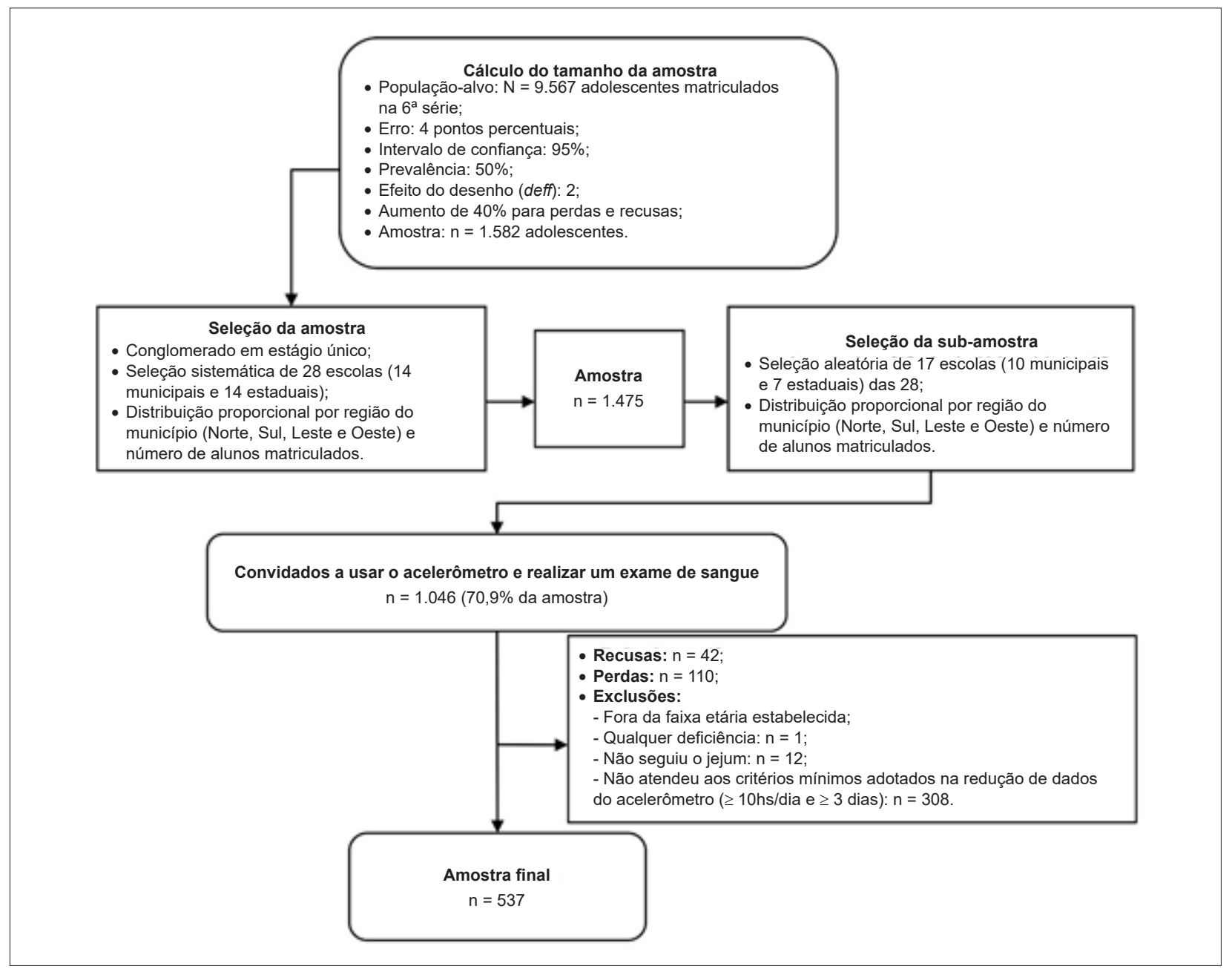

Figura 1 - Fluxograma do processo de amostragem do estudo 
medidas foram tomadas em triplicata pelo mesmo avaliador e, para fins de resultado final, adotou-se o valor médio das três medidas. $\mathrm{O}$ estado nutricional foi determinado por meio do IMC (massa corporal [kg] / estatura [m]²) e foi classificado de acordo com os critérios da World Health Organization (WHO) em baixo peso, peso normal, sobrepeso e obesidade. ${ }^{35}$

As amostras de sangue foram coletadas por técnicas em enfermagem, no período da manhã, e todos os adolescentes seguiram um jejum de, pelo menos, 12 horas. As concentrações de glicose $(\mathrm{mg} / \mathrm{dL})$, triglicerídeos $(\mathrm{mg} / \mathrm{dL})$, colesterol total $(\mathrm{mg} / \mathrm{dL})$ e lipoproteína de alta densidade (HDL-C; mg/dL) foram determinadas a partir do analisador bioquímico automático Labmax 240 premium, do fabricante Labtest, e determinadas pelo método de turbidimetria. A lipoproteína de baixa densidade (LDL-c) foi estimada pela equação de Friedewald, Levy e Fredrickson. ${ }^{36}$

A pressão arterial foi mensurada por meio do monitor automático da marca Omron $\mathrm{HEM}^{\circledR}-7200$, em uma única visita, no braço direito, com os adolescentes na posição sentada e após 5 minutos de repouso. Esse instrumento apresentou satisfatórios níveis de validade em amostra de adolescentes com faixa etária similar à do presente estudo. ${ }^{37}$ Foram realizadas três medidas (pressão sistólica $-\mathrm{CCl}=0,90$; IC95\%: 0,89 - 0,91 e diastólica - CCI = 0,80; IC95\%: 0,78 $-0,82)$, com intervalo de um minuto entre elas e utilizado o valor médio como resultado final.

O tempo em comportamento sedentário e atividade física moderada a vigorosa e o número de breaks foram mensurados por acelerômetros GT3X+ da ActiGraph ${ }^{\circledR}$. Os adolescentes foram orientados a utilizá-lo durante 7 dias consecutivos, fixado à cintura por um cinto elástico, no lado direito, retirando-o apenas para dormir, tomar banho, realizar atividades de lutas com quedas e aquáticas. A redução dos dados foi realizada no programa Actilife 6.12 e foram adotados os seguintes critérios: ${ }^{38}$ epoch de 15 segundos (reintegrados a 60 segundos); tempo de não uso $\geq 60$ minutos consecutivos de counts iguais a zero; uso por, no mínimo, 10 horas por dia, durante 3 dias ou mais dias, sendo pelo menos um de fim de semana.

O tempo em comportamento sedentário e de atividade física de intensidade moderada a vigorosa foi determinado com base nos limiares de Evenson et al.: ${ }^{38} \leq 100$ e > 2.295 counts/ minuto, respectivamente. Um break foi operacionalmente definido como o número de ocasiões em que o acelerômetro registrou 100 counts ou mais, por pelo menos um minuto. ${ }^{39}$

O número médio de breaks por dia foi determinado como segue: número médio de breaks em dias de semana (segunda a sexta-feira), multiplicado por cinco, e em dias de final de semana (sábado e domingo), multiplicado por dois, dividindo-se o somatório desses valores por sete. Esse mesmo procedimento foi aplicado para estimar a média ponderada do tempo de atividade física moderada a vigorosa e comportamento sedentário.

A exposição simultânea ao comportamento sedentário e ao número diário de breaks foi operacionalizada da seguinte maneira: a) tempo em comportamento sedentário categorizado em $<8$ horas/dia e $\geq 8$ horas/dia (tempo excessivo em comportamento sedentário) - este ponto de corte foi adotado por ter sido associado a piores indicadores de saúde cardiometabólica em adultos ${ }^{40}$ e não existir um ponto de corte bem estabelecido para adolescentes; b) número de breaks por dia em $<100$ breaks/dia e $\geq 100$ breaks/dia. Tal classificação foi estabelecida de acordo com curvas ROC [Receiver Operating Characteristic Curve], tendo em vista que não existe um ponto de corte definido para o número de breaks que demonstre maior risco ou proteção à saúde cardiometabólica, e pelo fato de que a quantidade de 100 breaks por dia apresentou valores mais equilibrados de sensibilidade e especificidade. Com base nisso, foram formados quatro grupos de adolescentes: 1) $\geq 8$ horas de comportamento sedentário e $<100$ breaks/dia; 2) $\geq 8$ horas de comportamento sedentário e $\geq 100$ breaks/dia; 3 ) $<8$ horas de comportamento sedentário e $<100$ breaks/dia; 4) $<8$ horas de comportamento sedentário e $\geq 100$ breaks/dia.

Foram considerados como perda amostral os adolescentes que não retornaram o termo de consentimento livre e esclarecido ou que se ausentaram da escola em pelo menos três visitas para a coleta dos dados. Os critérios de exclusão adotados foram: adolescentes que estavam fora da faixa etária de interesse do estudo (abaixo de 10 e acima de 14 anos); tinham alguma deficiência que os impedissem ou limitassem de praticar atividade física e/ou de responder ao questionário; não atenderam aos critérios adotados na redução de dados do acelerômetro; e relataram não ter seguido o jejum de pelo menos 12 horas.

\section{Análise estatística}

Para descrever as variáveis contínuas, foi utilizada a média e o desvio padrão naquelas com distribuição normal, a mediana e o intervalo interquartil para as que não apresentaram distribuição normal; e distribuição de frequência absoluta (n) e relativa (\%) para as categóricas. Foi utilizado o teste de Kolmogorov-Smirnov para verificar se os dados tinham aderência à distribuição normal. Para as variáveis categóricas, foi utilizado o teste do Qui-quadrado e, nas contínuas, os testes T de Student para amostras independentes (variáveis com distribuição normal) e U de Man-Whitney (variáveis sem distribuição normal) para comparar as variáveis entre os adolescentes incluídos e os excluídos das análises.

A regressão linear bruta e ajustada foi utilizada para analisar as associações entre o número de breaks por dia em comportamentos sedentários e marcadores cardiometabólicos e se elas eram moderadas pelo estado nutricional e o tempo excessivo em comportamento sedentário. Foram criados modelos de análise para cada variável dependente: glicose $(\mathrm{mg} / \mathrm{dL})$; colesterol total (mg/dL); triglicerídeos (mg/dL); HDL-c $(\mathrm{mg} / \mathrm{dL}), \mathrm{LDL}-\mathrm{c}(\mathrm{mg} / \mathrm{dL})$, pressão arterial sistólica $(\mathrm{mmHg})$, diastólica $(\mathrm{mmHg})$ e IMC $\left(\mathrm{kg} / \mathrm{m}^{2}\right)$. Em todos os modelos de análise, a variável independente foi o número médio de breaks diário em comportamento sedentário.

As covariáveis analisadas foram: sexo (masculino $=0$ e feminino $=1)$; idade (em anos); classe econômica $(A / B=0$ e $C / D / E=1)$; cor da pele (branco $=0$ e não branco $=1$ ); escolaridade da mãe (fundamental incompleto $=0$, fundamental completo $=1$ e médio completo ou mais $=2$ ); horas de sono (horas/dia); consumo de lipídios (g), total de gorduras 
saturadas (g), colesterol (mg), sódio (mg) e fibras (g); tempo de uso do acelerômetro (minutos/dia) e de atividade física de intensidade moderada-vigorosa (minutos/dia), de comportamento sedentário (minutos/dia) e IMC, exceto quando esta variável foi tratada como um marcador cardiometabólico no modelo foi tratado como variável dependente.

O método de seleção para entrada das variáveis no modelo ajustado foi o Foward, permanecendo aquelas que contribuíram para a redução nos valores dos resíduos, aumentaram o valor de $\mathrm{R}^{2}$ ajustado do modelo e modificaram em pelo menos $10 \%$ os valores dos coeficientes beta da regressão da variável número de breaks por dia. A qualidade de ajuste dos modelos foi avaliada a partir dos valores do fator de inflação de variância (VIF) (valores $<5$ indicaram ausência de multicolinearidade), distribuição dos resíduos em forma gráfica e homogeneidade das variâncias dos mesmos (Teste de Cook-Weisberg, valores de $p \geq 0,05$ indicaram presença de homocedasticidade).

Para testar a possível moderação do IMC e do comportamento sedentário na associação entre número de breaks por dia e os marcadores cardiometabólicos, foram criados os seguintes termos de interação: a) número de breaks/dia*comportamento sedentário $(<8$ horas e $\geq 8$ horas); b) número de breaks/dia*IMC ("sem excesso de peso" e "com excesso de peso corporal"). Esses termos foram incluídos nos modelos ajustados e considerados como interação presente quando o valor de $p$ foi $<0,05$. Neste caso, os modelos serão tratados separadamente de acordo com a classificação do comportamento sedentário $(<8$ horas e $\geq 8$ horas) e do IMC ("sem excesso de peso" [baixo peso + peso normal] e "com excesso de peso corporal" [sobrepeso + obesidade]).

Para comparar os valores médios de cada marcador cardiometabólico entre a exposição combinada a comportamento sedentário ( $<8$ horas e $\geq 8$ horas) e número diário de breaks (<100 breaks/dia e $\geq 100$ breaks/dia), foi utilizado o teste de Wald. Nesta análise, foram consideradas as médias de cada marcador cardiometabólico ajustadas pelas mesmas covariáveis dos modelos de regressão. As análises estatísticas foram realizadas no Stata 14.0 e o nível de significância adotado foi de $p<0,05$.

\section{Resultados}

Foram analisados dados de 537 adolescentes de 10 a 14 anos de idade (perdas, recusas e exclusões totalizaram 509 casos [48,6\% dos convidados] - Figura 1). O cálculo realizado a posteriori indicou que, com um tamanho de efeito (effect size) igual ou superior a 0,05 , alfa $(\alpha)$ de $5 \%$ e até 12 preditores no modelo, a amostra do presente estudo teve poder igual a $86 \%$.

Observou-se que não houve diferenças significativas $(p \geq 0,05)$ para as variáveis sexo, faixa etária, classe econômica, escolaridade da mãe e estado nutricional entre adolescentes que fizeram parte da amostra e da subamostra (dados não apresentados em tabela). Na comparação entre as características dos adolescentes incluídos e excluídos das análises, observou-se maior proporção de adolescentes entre 12 a 14 anos de idade, mães de menor escolaridade, com menores valores de breaks por dia, tempo em comportamento sedentário, menor consumo de gordura saturada, maior de lipídios e de sódio nos adolescentes excluídos das análises. Para as demais variáveis, não foram identificadas diferenças significativas $(p \geq 0,05)$ - Tabela 1 .

A maioria dos adolescentes era do sexo feminino, de 10 a 11 anos de idade, com cor da pele não branca, pertencia à classe econômica $\mathrm{C} / \mathrm{D} / \mathrm{E}$, filhos de mães com pelo menos o ensino fundamental completo e pouco mais de um terço tinha excesso de peso corporal. O tempo de atividade física, comportamento sedentário e número de breaks despendidos pelos adolescentes foi de respectivamente, 29,1; 451,0 e 100,3 (Tabela 1).

No modelo bruto, houve associação significativa entre número médio de breaks por dia e LDL-c $(\mathrm{p}=0,030)$, pressão arterial sistólica $(p=0,006)$ e IMC ( $p<0,001)$. Na análise ajustada, apenas associação entre o número médio de breaks por dia e o IMC ( $p<0,001)$ permaneceu estatisticamente significativa. O comportamento sedentário e o IMC não moderaram a associação entre número de breaks por dia e marcadores cardiometabólicos (Tabela 2). Os modelos finais alcançaram boa qualidade de ajuste: ausência de multicolinearidade (VIF entre 1,03 e 3,39), presença de homocedasticidade (teste de Cook-Weisberg com valores de $p$ variando de 0,054 a 0,335) e distribuição normal nos resíduos da regressão.

Os resultados do teste de Wald indicaram não existir diferenças significativas nos valores médios dos marcadores cardiometabólicos entre os adolescentes expostos a $\geq 8$ horas de comportamento sedentário e $<100$ breaks/dia, $\geq 8$ horas de comportamento sedentário e $\geq 100$ breaks/dia, $<8$ horas de comportamento sedentário e $<100$ breaks/dia e $<8$ horas de comportamento sedentário e $\geq 100$ breaks/dia (Figuras 2 e 3).

\section{Discussão}

Os resultados deste estudo indicaram que os adolescentes com maior número de breaks por dia no tempo em comportamento sedentário apresentaram valores mais baixos de IMC. Contudo, as associações com os demais marcadores cardiometabólicos não foram significativas e nem moderadas pelo estado nutricional e tempo excessivo em comportamento sedentário.

Estudos com adultos demonstraram que maior ocorrência de breaks estava associada à redução dos efeitos deletérios à saúde cardiometabólica decorrentes do tempo despendido em comportamento sedentário. ${ }^{41}$ No entanto, em adolescentes, essa relação vem sendo observada apenas com indicadores de gordura corporal. ${ }^{2,6}$ A ausência de associação dos breaks com os marcadores cardiometabólicos pode estar relacionada ao fato de os adolescentes acumularem a maior parte do seu tempo diário em comportamento sedentário em blocos de até 5 minutos. ${ }^{1,14,16}$ A exposição a blocos de tempo curtos em comportamento sedentário pode minimizar a redução da atividade da enzima LPL (lipoproteína lipase) e contribuir para o aumento do gasto energético. Esses dois fatores estão relacionados com diminuição nos níveis sanguíneos de glicose e triglicerídeos e aumento de HDL-c. ${ }^{42}$

O tempo excessivo de comportamento sedentário não moderou a associação entre o número de breaks e 
Tabela 1 - Comparação das características sociodemográficas, estado nutricional, consumo alimentar, horas de sono, marcadores cardiometabólicos, prática de atividade física, comportamento sedentário e número de breaks dos adolescentes incluídos e excluídos da análises (João Pessoa, Paraíba, 2014)

\begin{tabular}{|c|c|c|c|c|c|c|}
\hline \multirow{3}{*}{ Variáveis } & & \multirow{2}{*}{\multicolumn{2}{|c|}{$\begin{array}{c}\text { Incluídos nas análises } \\
\qquad(\mathrm{n}=537)\end{array}$}} & \multirow{2}{*}{\multicolumn{2}{|c|}{$\begin{array}{c}\text { Excluídos das análises } \\
\qquad(n=472)\end{array}$}} & \multirow{3}{*}{$\mathbf{p}^{*}$} \\
\hline & & & & & & \\
\hline & & $n$ & $\%$ & $\mathrm{n}$ & $\%$ & \\
\hline Sexo & & & & & & 0,281 \\
\hline Masculino & & 256 & 47,7 & 209 & 44,3 & \\
\hline Feminino & & 281 & 52,3 & 263 & 55,7 & \\
\hline Faixa etária & & & & & & $<0,001$ \\
\hline 10-11 anos & & 344 & 64,1 & 230 & 51,3 & \\
\hline 12-14 anos & & 193 & 35,9 & 242 & 48,7 & \\
\hline Classe econômica & & & & & & 0,614 \\
\hline$A / B$ & & 170 & 36,3 & 144 & 34,7 & \\
\hline $\mathrm{C} / \mathrm{D} / \mathrm{E}$ & & 298 & 63,7 & 271 & 65,3 & \\
\hline Cor da pele ${ }^{\S}$ & & & & & & 0,352 \\
\hline Branca & & 16 & 20,8 & 87 & 18,6 & \\
\hline Não branca & & 61 & 79,2 & 382 & 81,4 & \\
\hline Escolaridade da mãe $e^{\prime \prime}$ & & & & & & 0,010 \\
\hline Fundamental incompleto & & 148 & 33,5 & 166 & 41,9 & \\
\hline Fundamental completo & & 130 & 29,4 & 119 & 30,1 & \\
\hline Médio completo ou mais & & 164 & 37,1 & 111 & 28,0 & \\
\hline Índice de massa corporal (IMC) & & & & & & 0,085 \\
\hline Baixo peso & & 14 & 2,6 & 14 & 3,0 & \\
\hline Peso normal & & 326 & 61,4 & 321 & 68,6 & \\
\hline Sobrepeso & & 115 & 21,7 & 83 & 17,7 & \\
\hline Obesidade & & 76 & 14,3 & 50 & 10,7 & \\
\hline \multicolumn{7}{|l|}{ Exposição a comportamento sedentário } \\
\hline$<8$ horas/dia & & 343 & 63,9 & 192 & 66,9 & 0,386 \\
\hline \multirow[t]{2}{*}{$\geq 8$ horas/dia } & & 194 & 36,1 & 95 & 33,1 & \\
\hline & $\mathbf{n}$ & Média & DP & Média & DP & $\mathbf{p}^{\dagger}$ \\
\hline \multicolumn{7}{|l|}{ Variáveis comportamentais } \\
\hline Horas de sono (horas/dia) $)^{\S}$ & 536 & 9,7 & 1,6 & 9,6 & 1,6 & 0,871 \\
\hline Número de breaks (número/dia) & 537 & 100,3 & $91,5-108,3$ & 92,0 & $82,5-104,0$ & $<0,001$ \\
\hline Atividade física (minutos/dia)ף & 537 & 29,1 & $17,9-45,1$ & 30,5 & $16,5-47,0$ & $0,710^{\ddagger}$ \\
\hline Comportamento sedentário (minutos/dia) $)^{\pi}$ & 537 & 451,0 & $392,7-513,1$ & 432,8 & $377,0-500,7$ & $0,022^{\ddagger}$ \\
\hline Uso do acelerômetro (minutos/dia) & 537 & 855,3 & 94,9 & 816,0 & 109,7 & $<0,001$ \\
\hline \multicolumn{7}{|l|}{ Consumo alimentar } \\
\hline Lipídio (g) & 528 & 71,4 & 45,4 & 77,7 & 51,5 & 0,044 \\
\hline Gordura saturada $(\mathrm{g})^{\pi}$ & 528 & 15,0 & $8,0-23,0$ & 17,0 & $10,0-26,0$ & $0,001^{\ddagger}$ \\
\hline Sódio $(\mathrm{mg})^{\pi}$ & 528 & $2.055,5$ & $1.420,5-2.852,0$ & $2.161,0$ & $1.534,0-3.053,0$ & $0,028^{\ddagger}$ \\
\hline Fibras $(\mathrm{g})$ & 528 & 23,1 & 14,2 & 24,3 & 14,4 & 0,198 \\
\hline Colesterol (mg) & 528 & 176,8 & 190,4 & 188,5 & 240,2 & 0,397 \\
\hline \multicolumn{7}{|l|}{ Marcadores cardiometabólicos } \\
\hline IMC $\left(\mathrm{kg} / \mathrm{m}^{2}\right)$ & 531 & 19,5 & 4,0 & 19,5 & 3,6 & 0,410 \\
\hline PAS (mmHg) & 537 & 105,8 & 9,5 & 105,2 & 8,6 & 0,321 \\
\hline PAD (mmHg) & 537 & 62,4 & 7,0 & 61,9 & 6,9 & 0,318 \\
\hline Glicose (mg/dL) & 537 & 91,1 & 10,2 & 91,4 & 23,1 & 0,819 \\
\hline Colesterol $(\mathrm{mg} / \mathrm{dL})^{\S}$ & 536 & 159,4 & 31,7 & 158,1 & 32,1 & 0,580 \\
\hline Triglicerídeo $(\mathrm{mg} / \mathrm{dL})^{\pi}$ & 534 & 75,0 & $56-102$ & 73,0 & $54-98$ & $0,516^{\ddagger}$ \\
\hline $\mathrm{HDL}(\mathrm{mg} / \mathrm{dL})^{\S}$ & 536 & 43,9 & 9,5 & 43,4 & 9,3 & 0,463 \\
\hline LDL $(\mathrm{mg} / \mathrm{dL})^{\S}$ & 536 & 98,3 & 28,2 & 98,2 & 28,5 & 0,945 \\
\hline
\end{tabular}

DP: desvio padrão; *: teste qui-quadrado; †: T de Student para amostras independentes; ł: teste U de Mann-Whitney; §: variáveis com menor número de perdas ( $n=1)$; I/: variável com maior número de perdas ( $n=101)$; ף: dados apresentados como mediana e intervalo interquartil. 


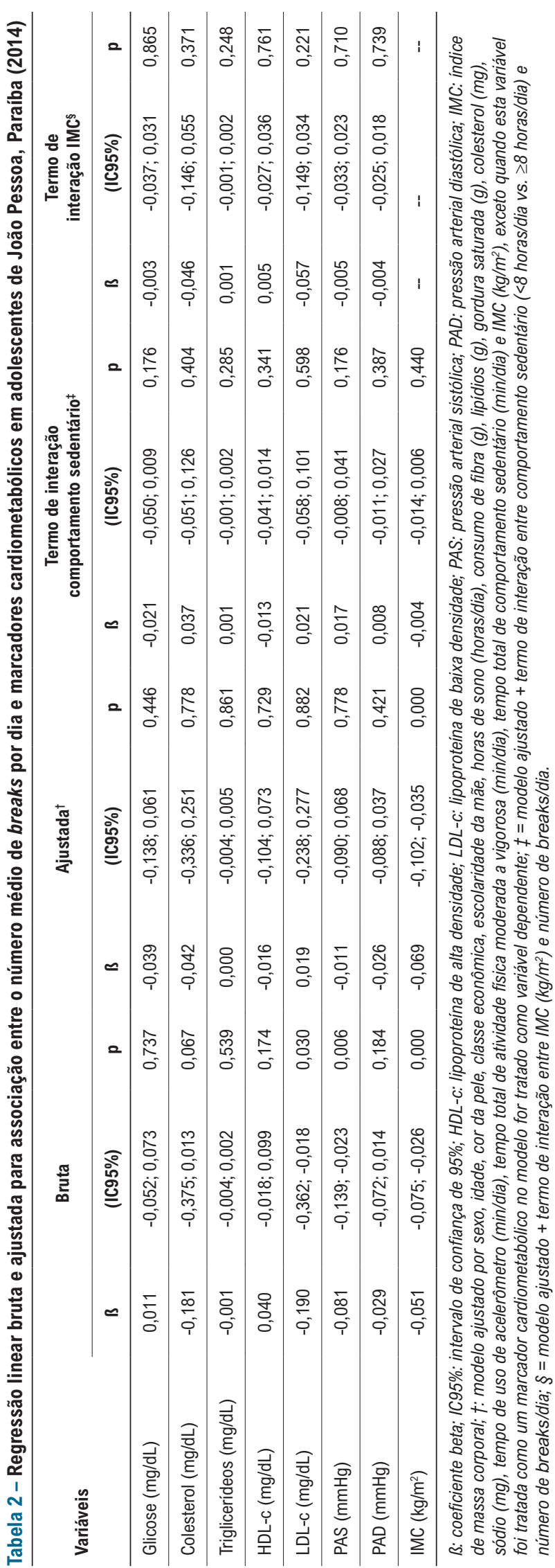

marcadores cardiometabólicos. Uma análise suplementar demonstrou que mais de $80 \%$ do tempo sedentário dos adolescentes do presente estudo foi acumulado em intervalos de tempo inferiores a 10 minutos, mesmo nos que tinham tempo excessivo em comportamento sedentário (dados não apresentados em tabela). Diante disso, é possível que os benefícios da inclusão de breaks sobre os marcadores cardiometabólicos sejam observados nos adolescentes expostos a longos períodos ininterruptos de comportamento sedentário.

Alguns estudos experimentais identificaram que a inclusão de breaks de intensidade moderada a vigorosa, com 3 minutos de duração e a cada meia hora, durante 3 horas de exposição a comportamentos sedentários, reduziu os níveis de insulina, peptídio $\mathrm{C}^{27,43}$ e de glicose. ${ }^{43}$ No entanto, esse resultado não foi confirmado por Sanders et al.,1 ao analisar os efeitos da inclusão de breaks de intensidade leve, com duração de 2 minutos e a cada 20 minutos, durante 8 horas de exposição a comportamentos sedentários. A inconsistência nos resultados desses estudos mostra que mais investigações são necessárias para suportar a hipótese de que os benefícios da inclusão de breaks ocorreriam em adolescentes expostos a tempos prolongados e ininterruptos de comportamento sedentário.

Uma possível menor resposta da LPL ao efeito hipotensor do comportamento sedentário e uma maior capacidade dos adolescentes para manter os marcadores cardiometabólicos em valores próximos aos considerados normais (homeostase), comparado aos adultos, são outras fontes de explicação para a ausência de associação entre breaks e marcadores cardiometabólicos nesse grupo populacional.

No presente estudo, verificou-se que adolescentes que tinham maior número de breaks apresentaram menores valores para o IMC, reforçando achados de outros estudos. ${ }^{2,6}$ Em termos de relevância clínica, o efeito dos breaks sobre o IMC foi de baixa magnitude (para cada break realizado, estima-se uma diminuição de $0,069 \mathrm{de} \mathrm{kg} / \mathrm{m}^{2}$ no IMC - effect size $=0,076)$. Apesar disso, a inclusão de breaks pode ser uma prática facilmente implementada no contexto de vida do adolescente, podendo ser uma dentre as várias ações a serem utilizadas em intervenções que visem à redução e/ou ao controle do IMC.

A inclusão de breaks no tempo sentado tende a promover aumento no gasto energético devido a maior prática de atividade física. Júdice et al., ${ }^{15}$ em estudo com adultos, observaram que a inclusão de um break resultava em aumento médio de 1,49 kcal/min no gasto energético. Nos adolescentes, os breaks podem gerar um gasto energético similar ao de adultos. Desse modo, a realização de 100 breaks ao longo do dia equivaleria a uma caminhada de cerca de 30 minutos de intensidade moderada. ${ }^{44}$ Maior tempo em comportamento sedentário está relacionado a um menor número de breaks e tempo de atividade física no lazer, ${ }^{45}$ e maior consumo de guloseimas, refrigerantes e alimentos industrializados/ultraprocessados. ${ }^{46}$ Desse modo, adolescentes que realizam mais breaks durante o dia teriam maior tempo de atividade física no lazer e menor consumo de alimentos ultraprocessados. Por ser um estudo transversal, não se pode descartar a possibilidade de que adolescentes com maior IMC teriam menor movimentação espontânea ao 

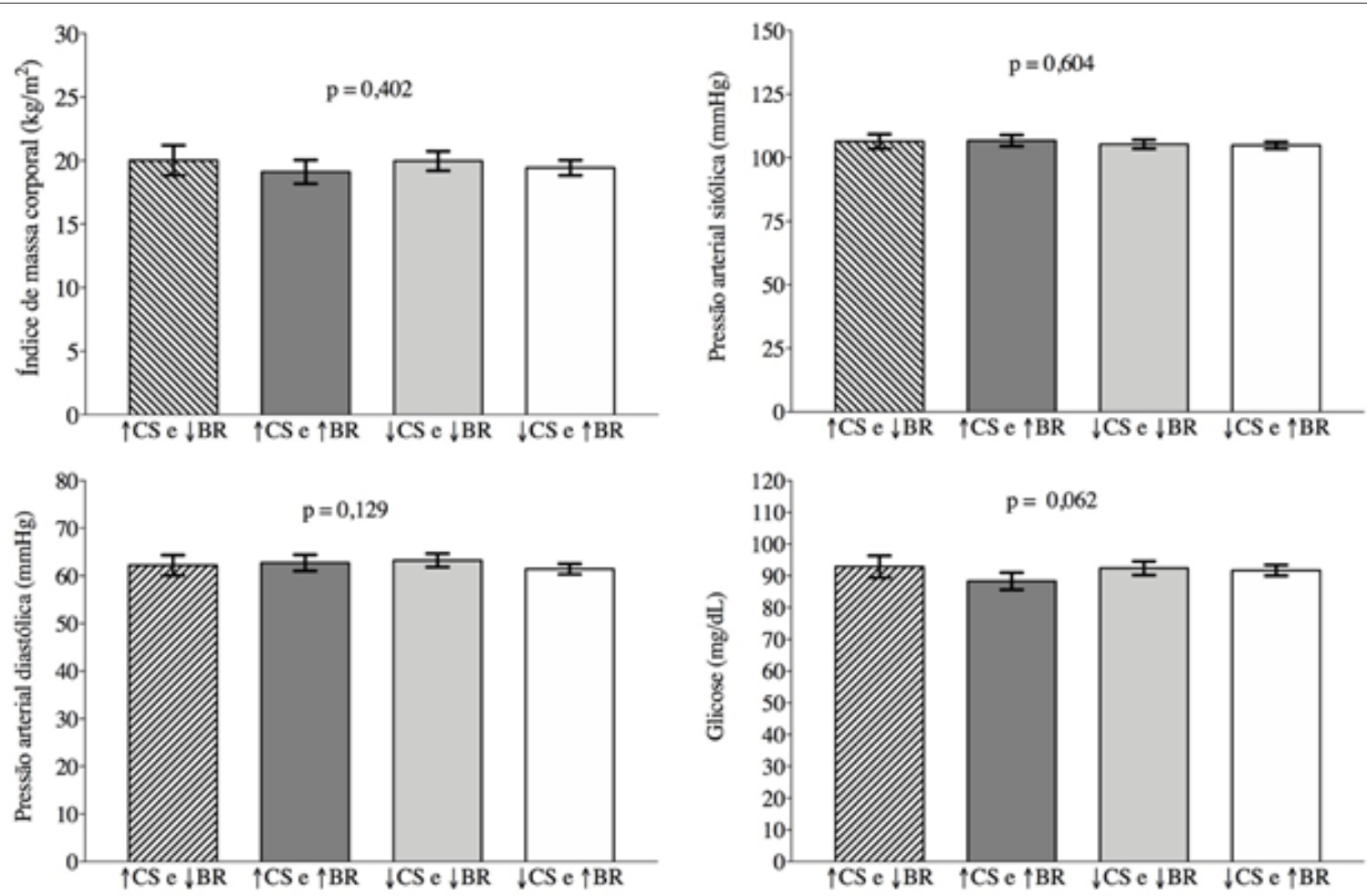

Figura 2 - Comparação dos valores médios de IMC, pressão arterial sistólica e diastólica e glicose entre exposição combinada a comportamento sedentário e breaks em adolescentes (João Pessoa, Paraiba, 2014). $\uparrow C S=\geq 8$ horas/dia; $\downarrow C S=<8$ horas/dia; $\uparrow B R=\geq 100$ breaks/dia; $\downarrow$ BR $=<100$ breaks/dia. CS: comportamento sedentário; BR: breaks.

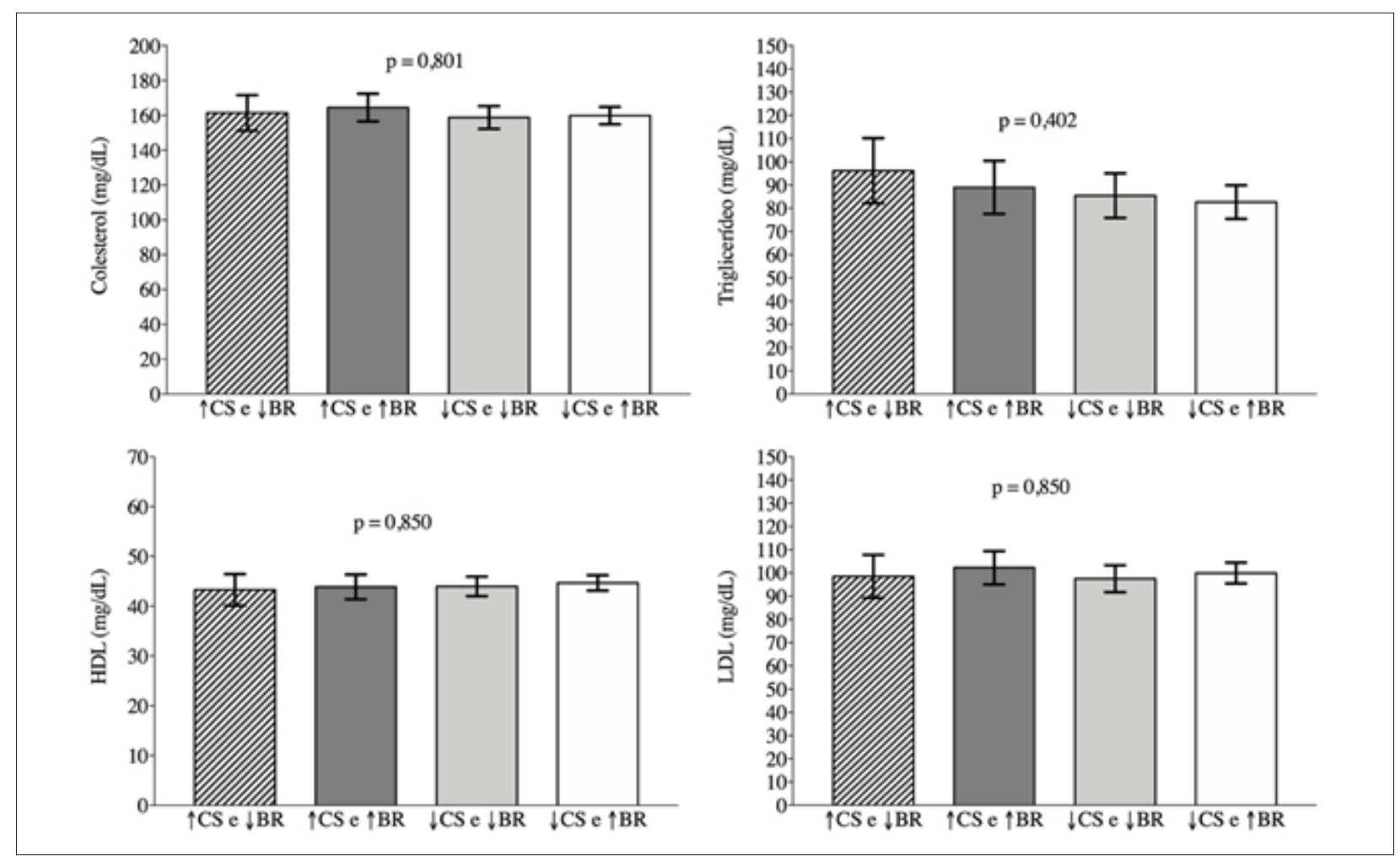

Figura 3 - Comparação dos valores médios de colesterol, triglicerídeos, HDL e LDL entre a exposição combinada a comportamento sedentário e breaks em adolescentes (João Pessoa, Paraiba, 2014). $\uparrow C S=\geq 8$ horas/dia; $\downarrow C S=<8$ horas/dia; $\uparrow B R=\geq 100$ breaks/dia; $\downarrow B R=<100$ breaks/dia; CS: comportamento sedentário; BR: breaks. 
longo dia, resultando em um menor número de breaks no comportamento sedentário.

Os pontos fortes deste estudo incluem: análise de dados em uma amostra representativa da população de escolares do sexto ano da rede pública de ensino de uma cidade do Nordeste do Brasil e poder suficiente para testar as hipóteses propostas; análise de diferentes marcadores cardiometabólicos; consideração, nas análises, de fatores de confusão importantes para a relação entre comportamento sedentário e marcadores cardiometabólicos (prática de atividade física, horas de sono, consumo alimentar).

As principais limitações deste estudo foram: ausência de mensuração da maturação sexual dos adolescentes, por ser uma variável que pode influenciar os marcadores cardiometabólicos ${ }^{47,48}$ e alguns tipos de comportamento sedentário; ${ }^{49}$ reintegração dos dados do acelerômetro de epochs de 15 para 60 segundos, o que pode ter subestimado o tempo em comportamento sedentário ${ }^{50} \mathrm{e}$ as magnitudes das associações; utilização do acelerômetro para mensurar o número de breaks em comportamento sedentário, uma vez que esse instrumento tem como base a medida da aceleração corporal e não tem capacidade de diferenciar de forma acurada a postura corporal (sentado, reclinado, em pé). ${ }^{51}$

\section{Conclusão}

Adolescentes com mais breaks por dia no tempo em comportamento sedentário tinham valores médios mais baixos para o IMC, mas não apresentaram diferenças nos valores dos demais marcadores cardiometabólicos bioquímicos (glicose, triglicerídeos, HDL-c, LDL-c, colesterol total e pressão arterial), independentemente do seu estado nutricional e do tempo excessivo de exposição ao comportamento sedentário.

\section{Agradecimentos}

Ao Conselho Nacional de Desenvolvimento Científico e Tecnológico (CNPq) e à Fundação de Apoio à Pesquisa do
Estado da Paraíba (FAPESQPB) pelo financiamento concedido para a realização da pesquisa.

\section{Contribuição dos autores}

Concepção e desenho da pesquisa: Lima NMM, Farias Júnior JC; Obtenção de dados: Lima NMM, Prazeres Filho A, Barbosa AO, Farias Júnior JC; Análise e interpretação dos dados e Análise estatística: Prazeres Filho A, Barbosa AO, Farias Júnior JC; Obtenção de financiamento: Farias Júnior JC; Redação do manuscrito: Lima NMM, Prazeres Filho A, Barbosa AO, Mendonça G, Farias Júnior JC; Revisão crítica do manuscrito quanto ao conteúdo intelectual importante: Mendonça G, Farias Júnior JC.

\section{Potencial conflito de interesse}

Não há conflito com o presente artigo

\section{Fontes de financiamento}

O presente estudo foi financiado pelo Conselho Nacional de Desenvolvimento Cientifico e Tecnológico - CNPq e Fundação de Apoio a Pesquisa - FAPESQ do Estado da Paraíba

\section{Vinculação acadêmica}

Este artigo é parte de dissertação de mestrado de Natália Maria Mesquita de Lima Quirino pela Universidade Federal da Paraíba (UFPB).

\section{Aprovação ética e consentimento informado}

Este estudo foi aprovado pelo Comitê de Ética em Pesquisa com Seres Humanos do Centro de Ciências da Saúde - UFPB sob o número de protocolo 240/13 - CAAE: 15268213.0.0000.5188. Todos os procedimentos envolvidos nesse estudo estão de acordo com a Declaração de Helsinki de 1975, atualizada em 2013. O consentimento informado foi obtido de todos os participantes incluídos no estudo.

\section{Referências}

1. Saunders TJ, Chaput J-P, Goldfield GS, Colley RC, Kenny GP, Doucet E, et al. Prolonged sitting and markers of cardiometabolic disease risk in children and youth: a randomized crossover study. Metabolism. 2013; 62(10):1423-8.

2. Carson V, Hunter S, Kuzik N, Gray CE, Poitras VJ, Chaput J-P, et al. Systematic review of sedentary behaviour and health indicators in school-aged children and youth: an update. Appl Physiol Nutr Me. 2016; 41(6):S240-S65

3. Vasankari V, Husu P, Vähä-Ypyä H, Suni J, Tokola K, Halonen J, et al. Association of objectively measured sedentary behaviour and physical activity with cardiovascular disease risk. Eur J Prev Cardiol. 2017; 24(12):1311-8.

4. Wu XY, Han LH, Zhang JH, Luo S, Hu JW, Sun K. The influence of physical activity, sedentary behavior on health-related quality of life among the general population of children and adolescents: A systematic review. PloS one. 2017; 12(11):e0187668-e.

5. Verswijveren SJ, Lamb KE, Bell LA, Timperio A, Salmon J, Ridgers ND. Associations between activity patterns and cardio-metabolic risk factors in children and adolescents: A systematic review. PloS one. 2018; 13(8):e0201947.
6. Fröberg A, Raustorp A. Objectively measured sedentary behaviour and cardiometabolic risk in youth: a review of evidence. EurJ Pediatr. 2014; 173(7):845-60.

7. Cliff DP, Hesketh KD, Vella SA, Hinkley T, Tsiros MD, Ridgers ND, et al Objectively measured sedentary behaviour and health and development in children and adolescents: systematic review and meta-analysis. Obes Rev. 2016; 17(4):330-44

8. Hamilton MT, Hamilton DG, Zderic TW. Exercise physiology versus inactivity physiology: an essential concept for understanding lipoprotein lipase regulation. Exerc Sport Sci Rev. 2004; 32(4):161-6

9. Hamilton MT, Hamilton DG, Zderic TW. Role of low energy expenditure and sitting in obesity, metabolic syndrome, type 2 diabetes, and cardiovascular disease. Diabetes. 2007; 56(11):2655-67.

10. Healy GN, Wijndaele K, Dunstan DW, Shaw JE, Salmon J, Zimmet PZ, et al. Objectively measured sedentary time, physical activity, and metabolic risk: The Australian Diabetes, Obesity and Lifestyle Study (AusDiab). Diabetes Care. 2008; 31(2):369-71.

11. Miles-Chan JL, Dulloo AG. Posture allocation revisited: breaking the sedentary threshold of energy expenditure for obesity management. Front Physiol. 2017; 8:420. 
12. Biddle SJH, Pearson N, Salmon J. Sedentary Behaviors and adiposity in young people: causality and conceptual model. Exerc Sport Sci Rev. 2018; 46(1):18-25.

13. Fletcher EA, Carson V, McNaughton SA, Dunstan DW, Healy GN, Salmon J. Does diet mediate associations of volume and bouts of sedentary time with cardiometabolic health indicators in adolescents? Obesity (Silver Spring, Md). 2017; 25(3):591-9

14. Ramos DE, Bueno MRO, Vignadelli LZ, Werneck AO, Ronque ERV, Coelho-E-Silva MJ, et al. Pattern of sedentary behavior in brazilian adolescents. Rev Bras Ativ Fis Saude. 2018; 23:1-6.

15. Júdice PB, Silva AM, Berria J, Petroski EL, Ekelund U, Sardinha LB. Sedentary patterns, physical activity and health-related physical fitness in youth: a cross-sectional study. Int J Behav Nutr Phy. 2017; 14(1):25.

16. Mendonça G, Prazeres Filho A, Barbosa AO, Farias Júnior JC. Padrões de comportamento sedentário em adolescentes de um município da região Nordeste do Brasil. Rev Bras Ativ Fis Saude. 2018; 23:1-9.

17. Tremblay MS, LeBlanc AG, Kho ME, Saunders TJ, Larouche R, Colley RC, et al. Systematic review of sedentary behaviour and health indicators in school-aged children and youth. Int J Behav Nutr Phys Act. 2011; 8:98.

18. Healy GN, Dunstan DW, Salmon J, Cerin E, Shaw JE, Zimmet PZ, et al. Breaks in sedentary time. D C. 2008; 31(4):661.

19. Hamilton MT, Healy GN, Dunstan DW, Zderic TW, Owen N. Too little exercise and too much sitting: Inactivity physiology and the need for new recommendations on sedentary behavior. Curr Cardiovasc Risk Rep. 2008; 2(4):292.

20. Wilson AN, Olds T, Lushington K, Petkov J, Dollman J. The impact of 10-minute activity breaks outside the classroom on male students' on-task behaviour and sustained attention: a randomised crossover design. Acta Paediatr. 2016; 105(4):e181-8.

21. Chastin SF, Egerton T, Leask C, Stamatakis E. Meta-analysis of the relationship between breaks in sedentary behavior and cardiometabolic health. Obesity. 2015; 23(9):1800-10.

22. Poitras VJ, Gray CE, Borghese MM, Carson V, Chaput J-P, Janssen I, et al. Systematic review of the relationships between objectively measured physical activity and health indicators in school-aged children and youth. Appl Physiol Nutr Me. 2016; 41 (6 (Suppl. 3)):S197-S239.

23. Carson V, Wong SL, Winkler E, Healy GN, Colley RC, Tremblay MS. Patterns of sedentary time and cardiometabolic risk among Canadian adults. Prev Med. 2014; 65:23-7.

24. Biddle SJH, Garcia Bengoechea E, Pedisic Z, Bennie J, Vergeer I, Wiesner G. Screen Time, Other Sedentary Behaviours, and Obesity Risk in Adults: A Review of Reviews. Curr Obes Rep. 2017; 6(2):134-47.

25. Carson V, Stone M, Faulkner G. Patterns of sedentary behavior and weight status among children. Pediatr Exerc Sci. 2014; 26(1):95-102.

26. Saunders TJ, Tremblay MS, Mathieu M-È, Henderson M, O'Loughlin J, Tremblay A, et al. Associations of sedentary behavior, sedentary bouts and breaks in sedentary time with cardiometabolic risk in children with a family history of obesity. PLoS ONE. 2013; 8(11):e79143.

27. Broadney MM, Belcher BR, Berrigan DA, Brychta RJ, Tigner IL, Shareef $\mathrm{F}$, et al. Effects of interrupting sedentary behavior with short bouts of moderate physical activity on glucose tolerance in children with overweight and obesity: A randomized crossover trial. Diabetes Care. 2018; 41(10):2220-8.

28. Colley RC, Garriguet D, Janssen I, Wong SL, Saunders TJ, Carson V, et al. The association between accelerometer-measured patterns of sedentary time and health risk in children and youth: results from the Canadian Health Measures Survey. BMC Public Health. 2013; 13(1):200.

29. Kelly AS, Barlow SE, Rao G, Inge TH, Hayman LL, Steinberger J, et al. Severe obesity in children and adolescents: identification, associated health risks, and treatment approaches: a scientific statement from the American Heart Association. Circulation. 2013; 128(15):1689-712.
30. Umer A, Kelley GA, Cottrell LE, Giacobbi P, Jr., Innes KE, Lilly CL. Childhood obesity and adult cardiovascular disease risk factors: a systematic review with meta-analysis. BMC Public Health. 2017; 17(1):683.

31. Brasil. Ministério do Planejamento Orçamento e Gestão. Pesquisa Nacional por Amostra de Domicílios (PNAD) 2011. Rio de Janeiro: Instituto Brasileiro de Geografia e Estatística (IBGE). 2012.

32. Pinheiro ABV, Lacerda EMA, Benzecry EH, Gomes MCS, Costa VM. Tabela para avaliação de consumo alimentar em medidas caseiras. $5^{\underline{a}}$ ed. São Paulo: Atheneu, 2008.

33. Verly-Jr E, Castro MA, Fisberg RM, Marchioni DML. Precision of usual food intake estimates according to the percentage of individuals with a second dietary measurement. J Acad Nutr Diet. 2012; 112(7):1015-20.

34. Trumbo P, Yates AA, Schlicker S, Poos M. Dietary reference intakes: vitamin $\mathrm{A}$, vitamin $\mathrm{K}$, arsenic, boron, chromium, copper, iodine, iron, manganese, molybdenum, nickel, silicon, vanadium, and zinc. J Am Diet Asso. 2001; 101(3):294-301.

35. World Health Organization Multicentre Growth Reference Study Group. WHO child growth standards. Methods and development: length/heightfor-age, weight-for-age, weight-for-length, weight-for-height and body mass index-for-age. . Disponivel em: http://www.who.int/childgrowth/ standards/technical_report/en/index.html. Acessado em 21/09/2015.

36. Friedewald WT, Levy RI, Fredrickson DS. Estimation of the concentration of low-density lipoprotein cholesterol in plasma, without use of the preparative ultracentrifuge. Clin Chem. 1972; 18(6):499-502.

37. Christofaro DGD, Fernandes RA, Gerage AM, Alves MJ, Polito MD, Oliveira AR. Validation of the Omron HEM 742 blood pressure monitoring device in adolescents. Arq Bras Cardiol. 2009; 92(1):10-5.

38. Evenson KR, Catellier DJ, Gill K, Ondrak KS, McMurray RG. Calibration of two objective measures of physical activity for children. J Sports Sci. 2008; 26(14):1557-65.

39. Altenburg TM, Chinapaw MJ. Bouts and breaks in children's sedentary time: currently used operational definitions and recommendations for future research. Prev Med. 2015; 77:1-3.

40. Owen N, Sparling PB, Healy GN, Dunstan DW, Matthews CE. Sedentary behavior: emerging evidence for a new health risk. Mayo Clin Proc. 2010; 85(12):1138-41.

41. Brocklebank LA, Falconer CL, Page AS, Perry R, Cooper AR. Accelerometer-measured sedentary time and cardiometabolic biomarkers: a systematic review. Prev Med. 2015; 76:92-102.

42. Ryan DJ, Stebbings G, Onambele G. The emergence of sedentary behaviour physiology and its effects on the cardiometabolic profile in young and older adults. Age. 2015; 37(5):89.

43. Belcher BR, Berrigan D, Papachristopoulou A, Brady SM, Bernstein SB, Brychta RJ, et al. Effects of interrupting children's sedentary behaviors with activity on metabolic function: a randomized trial. J Clin Endocrinol Metab. 2015; 100(10):3735-43.

44. Butte NF, Watson KB, Ridley K, Zakeri IF, McMurray RG, Pfeiffer KA, et al. A youth compendium of physical activities: activity codes and metabolic intensities. Med Sci Sports Exerc. 2018; 50(2):246-56.

45. Pearson N, Braithwaite R, Biddle SJ, van Sluijs EM, Atkin AJ. Associations between sedentary behaviour and physical activity in children and adolescents: a meta-analysis. Obes Rev. 2014; 15(8):666-75.

46. Costa CS, Flores TR, Wendt A, Neves RG, Assunção MCF, Santos IS Comportamento sedentário e consumo de alimentos ultraprocessados entre adolescentes brasileiros: Pesquisa Nacional de Saúde do Escolar (PeNSE), 2015. Cad Saude Publica. 2018; 34:e00021017.

47. Katon JG, Flores YN, Salmeron J. Sexual maturation and metabolic profile among adolescents and children of the Health Worker Cohort Study in Mexico. Salud Publica Mex. 2009; 51(3):219-26. 
48. Mascarenhas LP, Leite N, Titski AC, Brito LM, Boguszewski MC. Variability of lipid and lipoprotein concentrations during puberty in Brazilian boys. J Pediatr Endocrinol Metab. 2015; 28(1-2):125-31.

49. Piola TS, Bacil EDA, Silva MP, Campos JG, Neto NAM, Campos W. Comportamento sedentário em adolescentes: análise hierárquica de fatores associados. Revista Contexto Saúde. 2019; 19(37):128-36.
50. Banda JA, Haydel KF, Davila T, Desai M, Bryson S, Haskell WL, et al Effects of varying epoch lengths, wear time algorithms, and activity cutpoints on estimates of child sedentary behavior and physical activity from accelerometer data. PLoS One. 2016; 11(3):e0150534.

51. Stålesen J, Vik FN, Hansen BH, Berntsen S. Comparison of three activity monitors for estimating sedentary time among children. BMC Sports Sci Med Rehabilitation. 2016; 8(1):2. 